\title{
Blood Metabolomic Profiling Predicts Postoperative Gastrointestinal Function Of Colorectal Surgical Patients Under The Guidance Of Goal-Directed Fluid Therapy
}

\section{Tao Xie}

Changhai Hospital

Zhengyu Jiang

Naval Medical Center

Cen Wen

Changhai Hospital

\section{Du Shen}

Changhai Hospital

\section{Jinjun Bian}

Changhai Hospital

Shanshan Liu

Chenggong Hospital of Xiamen University

\section{Xiaoming Deng}

Changhai Hospital

Yanping Zha ( $\square$ zhayuxuan1128302@163.com )

Changhai Hospital https://orcid.org/0000-0002-0165-6576

\section{Research}

Keywords: colorectal surgery, metabolomics, goal-directed fluid therapy, gastrointestinal function

Posted Date: July 27th, 2020

DOl: https://doi.org/10.21203/rs.3.rs-46081/v1

License: (c) (1) This work is licensed under a Creative Commons Attribution 4.0 International License. Read Full License

Version of Record: A version of this preprint was published at Aging on March 10th, 2021. See the published version at https://doi.org/10.18632/aging.202711. 


\section{Abstract}

Background: Postoperative gastrointestinal function influences postoperative recovery and hospital stay length for patients undergoing colorectal surgery. Goal-directed fluid therapy (GDFT) restricts fluid administration to an amount required to prevent dehydration. Although the fluid management of GDFT could decrease the incidence of postoperative complications in patients who undergo high-risk surgery, certain patients may not respond to GDFT. Thus, to achieve optimal treatment, identification of patients suitable for GDFT is necessary. Accordingly, in this study, we evaluated the predictive capacity of metabolomics profiling for postoperative recovery after surgery for colorectal cancer.

Methods: Metabolomic profiling of 48 patients with colorectal cancer who underwent surgery was performed. Patients were divided into delayed-and enhanced-recovered groups based on gastrointestinal function within 72 hours, and the results of -omics analysis showed differential serum metabolites between the two groups of patients in the postanesthesia care unit 24 hours after surgery. A support vector machine model was applied to evaluate the curative effects of GDFT in different patients.

Results: Four metabolites, oleamide, ubiquinone-1, acetylcholine, and oleic acid, were found to be highly associated with postoperative gastrointestinal function and could serve as potential biomarkers. Moreover, four pathways were found to be highly related to postoperative gastrointestinal recovery. Among these, the vitamin B6 metabolism pathway may be a common pathway for improving postoperative recovery in various diseases.

Conclusion: Our findings proposed a novel method to predict postoperative recovery of gastrointestinal function based on metabolomic profiling and suggested the potential mechanisms contributing to gastrointestinal function after surgical resection of colorectal cancer under the fluid management of GDFT.

Trial registration: Chinese Clinical Trial Registry, ChiCTR1800015899, Registered 27 April 2018, http://www.chictr.org.cn/showproj.aspx?proj=26623

\section{Background}

Postoperative gastrointestinal (GI) recovery significantly influences peri-operative complications and prognosis in patients undergoing colorectal surgery, particularly for elderly patients who are at risk of increased hospital stay and organ dysfunction owing to delayed GI recovery. ${ }^{[1]}$ The amount of intravenous (i.v.) fluid administration is related to peri-operative morbidity, and excess or insufficient fluid delivery can increase postoperative complications.[2, 3] Traditional i.v. therapy is usually determined by the perceived magnitude of surgical trauma without the supporting physiological principles.[4] Therefore, i.v. therapy might be harmful if patients do not respond to fluid delivery or do not exhibit enhanced blood flow upon fluid administration.[5] Goal-directed fluid therapy (GDFT) based on the Enhanced Recovery After Surgery (ERAS) program restricts fluid administration to an amount required to prevent dehydration, 
both during and after surgery.[6, 7] Studies have shown that GDFT decreases the incidence of postoperative complications in patients who undergo high-risk surgery.[8,9]

By adjusting arterial pressure and blood flow parameters, including cardiac output, cardiac index, stroke volume index, stroke volume (SV) variation (SVV), and oxygen delivery, GDFT can modify peri-operative fluid administration to achieve suitable end-organ perfusion.[7] SVV is used to reflect variations in left ventricular output secondary to intrathoracic pressure changes induced by mechanical ventilation and represents the percentage of change between the maximum and minimum SVs over a period divided by their average value. SVV has been shown to be an accurate predictor of fluid responsiveness[10] and could be used as a resuscitation endpoint to evaluate postoperative recovery.

The ERAS program has facilitated progress in promoting the recovery of intestinal function and has been proposed as a standard strategy for enhancement of postoperative recovery in northern Europe and other countries.[11, 12] However, few studies have established prediction and risk assessments of postoperative GI function for colorectal surgery patients, and it is usually only by clinical outcomes that could evaluate whether the postoperative GI function recovery of patients are favorable. Therefore, it is necessary to identify measures that could predict the possible long-term postoperative recovery condition of GI function in patients at immediately timepoint or early after surgery, from which early intervention strategies can be conducted in patients with predicted poor recovery condition of GI function.

Metabolomics is a novel approach with many applications in biological research. With the development of advanced analytical techniques and bioinformatics, metabolomics has been extensively applied as a holistic diagnostic tool in clinical and biomedical studies. In contrast to proteomics, genomics, and other methods that reflect indirect changes in the system, metabolomics reveals the direct response of the physiological environment.[13] In the case of postoperative recovery of intestinal function, which develops asymptotically, metabolite changes can occur much earlier in the postoperative period. Metabolomics can reflect the influence of surgical trauma and anesthesia on the general condition of patients during the early postoperative period. However, whether metabolic factors affect the recovery of intestinal function or its predictive effect on postoperative recovery after colorectal surgery has not been extensively studied.

Recently, researchers have demonstrated that some patients exhibit poor postoperative recovery condition under the intraoperative fluid management of GDFT.[14] Therefore, in this study, we aimed to further identify the influence of GDFT on the recovery of postoperative GI function in patients who underwent colorectal resection, and to explore the potential biomarkers that can predict the possible longterm recovery condition of $\mathrm{Gl}$ function in patients by metabolomics analysis of plasma samples early after surgery.

\section{Methods}

\section{Study design and participants}


This was a single-center, prospective, observational cohort study. Two parallel groups (enhanced and delayed recovery) were enrolled according to the postoperative time of flatus. Patients who had undergone elective surgery for colorectal cancer resection were enrolled from December 2018 to October 2019 at Shanghai Changhai Hospital, Second Military Medical University. Written informed consent was obtained from all participants on the day before surgery. Patients who met any of the following criteria were excluded: ASA IV or V; age < 18 years or > 70 years; fasting time < 8 hours or > 20 hours; preoperative ileus; body mass index $(\mathrm{BMI})<18 \mathrm{~kg} / \mathrm{m}^{2}$ or $>25.0 \mathrm{~kg} / \mathrm{m}^{2}$; presence of cardiovascular diseases, such as coronary artery or valvular diseases, New York Heart Association Functional Classification class II or higher heart failure, and arrhythmia; presence of metabolic diseases, such as severe diabetes, ketoacidosis, hyperosmolar syndrome, or long-term hypoglycemia; presence of systemic immune disease; presence of liver and kidney dysfunction; presence of chronic obstructive pulmonary disease; and refusal to participate in the study or presence of mental illness and inability to communicate correctly. In addition, because of the pneumoperitoneum in laparoscopic surgery may affect the change of chest pressure, and the accuracy of SVV monitoring may be influenced, thereby the laparoscopic colorectal surgeries were also excluded.

\section{Anesthesia}

General anesthesia was induced by midazolam (0.04 mg/kg), propofol (1-2.5 mg/kg), fentanyl (2.5-6 $\mu \mathrm{g} / \mathrm{kg})$, cis-atracurium (0.2-0.3 mg/kg), and dexamethasone (10 mg). Anesthesia was maintained by inhalation of sevoflurane (0.8-1.3 MAC) with intermittent supplementation using other auxiliary drugs, such as remifentanyl $(0.1-0.2 \mu \mathrm{g} / \mathrm{kg} / \mathrm{min})$, dexmedetomidine $(0.2-0.5 \mu \mathrm{g} / \mathrm{kg} / \mathrm{h})$, cis-atracurium, and fentanyl. To accelerate postoperative extubation, inhalation was discontinued, and total intravenous anesthesia by propofol (100-200 mg/kg/min) was applied before abdominal closure. Patients were ventilated in a volume-controlled mode with a tidal volume of $8 \mathrm{~mL} / \mathrm{kg}$ (air: 40-100\% oxygen). The respiratory rate was set to maintain the $\mathrm{PaCO}_{2}$ between 35 and $45 \mathrm{mmHg}$ to improve the operating conditions, and the positive end-expiratory pressure was set at $6-8 \mathrm{~mL} \mathrm{H}_{2} \mathrm{O}$ when necessary. For intraoperative analgesia, each patient received bilateral transverse abdominal fascia nerve blocks containing $20-30 \mathrm{~mL}$ ropivacaine $(0.375 \%)$ after induction.

\section{Fluid management and hemodynamic monitoring}

On arrival at the operating room, patients received intravenous ringer lactate $(5 \mathrm{~mL} / \mathrm{kg})$, and hemodynamic parameters were recorded as the baseline. After induction of anesthesia, a 20-G radial arterial line was inserted and connected to an electrocardiogram monitor and fourth-generation Vigileo/Flotrac system (Edwards Lifesciences, Irvine, CA, USA) to determine changes in arterial blood pressure waveforms, SVVs, and cardiac indexes. A double-lumen 5.5-Fr catheter (arrow central venous catheter; Teleflex Life Sciences Ltd., Athlone, Ireland) was inserted into the right internal jugular vein for colloid supplementation. A continuous infusion of ringer lactate was given through peripheral veins during the operation at a rate of $3 \mathrm{~mL} / \mathrm{kg} / \mathrm{h}$. Colloid (hydroxyethyl starch 130/0.4 sodium chloride injection; Fresenius Kabi, USA) management was conducted under the guidance of the Vigileo/Flotrac 
system. If the SVV changed by more than 10\% 2-3 consecutive times, the colloid was injected via the central vein at $4 \mathrm{~mL} / \mathrm{kg}$. After $5 \mathrm{~min}$, SVV changes were observed again. Vasoactive agents were given according to changes in intra-operative hemodynamics based on the recommendations of the anesthesiologist. Transfusions were applied according to recent guidelines[15].

\section{Data collection}

The study was designed as a two-arm, parallel, prospective, observational cohort. The primary endpoint of the study was the timepoint of flatus and differences in blood metabolomics between the two groups. Hemodynamic parameters at different timepoints, surgical resection methods, total intra-operative time, and peri-operative fluid volume were recorded. Additionally, the number of hemodynamic fluctuations, such as hypotension or hypertension, was collected. The timepoints of flatus and feeding were recorded, and the time of flatus was used as an indicator of postoperative recovery. The occurrence of flatus 72 hours after surgery was identified as delayed postoperative recovery. The length of hospital stay and duration of postoperative complications were calculated.

\section{Blood sample collection}

Blood samples were collected from all patients at admission to the postanesthesia care unit (PACU) and at 24 hours after surgery. Blood was collected into heparinized tubes, and plasma was immediately separated by centrifugation at $3000 \mathrm{rpm}$ for $10 \mathrm{~min}$ and stored at $-80^{\circ} \mathrm{C}$ until metabolomics analysis.

\section{Liquid chromatography (LC)/mass spectrometry (MS) analysis}

LC/MS analysis was carried on an Ultimate 3000LC Q Exactive platform (Thermo, USA). The parameters for electron spray ionization (ESI)+ and ESI- ion modes were as follows: heater temperature, $300{ }^{\circ} \mathrm{C}$; sheath gas flow rate, 45 arb; auxiliary gas flow rate, 15 arb; sweep gas flow rate, 1 arb; spray voltage, 3.0 kV; capillary temperature, $350{ }^{\circ} \mathrm{C}$; S-Lens RF level, $30 \%$. The data were analyzed using feature extraction and preprocessed with Compound Discoverer software (Thermo). In total, 2015 features in ESI+ ion mode and 1601 features in ESI- ion mode were evaluated, and editing of data was performed with the Multivariate Analysis (MVA) feature of SIMCA-P software (Umetrics AB, Umea, Sweden).

\section{Biomarker identification}

A supervised learning model was built by a support vector machine (SVM) to classify the delayed and enhanced postoperative recovery groups. Finally, we used radial as SVM kernel function under type C classification and selected 22 features $(A-B)$ and 10 features $(C-D)$ to construct the two SVM models separately. Under these parameters, the predictive accuracies reached $92.8 \%$ and $88.8 \%$, and the area under the curve (AUC) values were 0.983 and 0.941 for groups $A-B$ and $C-D$, respectively, indicating that the model was effective.

\section{Statistical analysis}


Statistical analysis was performed using GraphPad Prism version 8.0 (San Diego, CA, USA). Baseline demographics and postoperative complications were evaluated using Pearson's chi-square tests for categorical variables, with Fisher's exact test for small counts. Analysis of variance with Tukey's correction was used for continuous data. Results with $P$ values less than 0.05 were considered statistically significant, and all data were analyzed using intention-to-treat principles.

\section{Results}

\section{Basic characteristics of patients in the delayed and enhanced postoperative recovery groups}

The demographic data for all patients were shown in Table 1. Among 48 patients with colorectal cancer, 24 showed enhanced postoperative recovery after surgery. No significant differences in age, six, BMI, ASA grade, operation duration, pre-operative SVV, postoperative SVV, pre-operative mean blood pressure (MBP), postoperative MBP, intra-operative fluid administration, or 24-hours peri-operative fluid administration was observed between the enhanced and delayed groups. The enhanced group showed earlier times to flatus, defecation, and feeding and decreased occurrence of anemia, and postoperative nausea and vomiting.

\section{Overall metabolomics analysis of blood samples}

The metabolomics of 96 blood samples from 48 patients who underwent surgery for colorectal cancer in the enhanced and delayed groups were characterized and compared. Blood samples were collected when patients were in the PACU and at 24 hours after surgery for all 48 patients. Delayed PACU samples were separated as group A, enhanced PACU samples were separated as group B, delayed 24 hours samples were separated as group C, and enhanced PACU samples were separated as group D. Representative ESI+ and ESI- ion chromatograms for each group are presented in Supplementary Figure 1. In total, 4,652 molecular features in ESI+ ion mode and 1,898 molecular features in ESI- ion mode were obtained and subjected to MVA. Principal component analysis extracted 16 principal components for ESI+ ion mode with a $Q^{2}$ of 0.255 and $R^{2} X$ of 0.553 . For ESI- ion mode (Figure $2 A$ ), there were 15 principal components, with a $Q^{2}$ of 0.284 and $R^{2} X$ of 0.584 (Figure $2 B$ ). From the overall point of view, enhanced and delayed group distributions were overlapped in both ESI+ ion mode and ESI- ion mode, and a significant difference existed between PACU and 24 hours after surgery groups.

The partial least squares discriminant analysis (PLS-DA) results of enhanced and delayed postoperative recovery samples in the PACU revealed differences in their distributions, with a $Q^{2}$ of 0.343 and $R^{2} Y$ of 0.97 in ESI+ ion mode (Figure $1 \mathrm{~A}$ ) and a $\mathrm{Q}^{2}$ of 0.433 and $\mathrm{R}^{2} \mathrm{Y}$ of 0.95 in ESI- ion mode (Figure 1B). Although the $R^{2} Y$ reflected a not over lifting model, the $Q^{2}$ had low prediction rate, suggesting that this model may be unreliable. Features with variable importance in projection (VIP) scores greater than 1.0 in multivariate statistical analysis and $p$ values of less than 0.05 in univariate analysis were considered as the most significant metabolites. Twenty metabolites for ESI+ ion mode and eight metabolites for ESI-ion mode were selected as the most significant differentiating metabolites (Supplemental Table 1). 
The PLS-DA results for blood samples collected 24 hours after surgery in the enhanced and delayed postoperative recovery patients revealed differences in their distributions, with a $Q^{2}$ of 0.691 and $R^{2} Y$ of 0.985 for ESI+ ion mode (Figure 1C) and a $Q^{2}$ of 0.453 and $R^{2} Y$ of 0.811 for ESI- mode (Figure 1D). This model also appeared to have a low prediction rate and to be not over lifting. Features with VIP scores greater than 1.0 in multivariate statistical analysis and $p$ values less than 0.05 in univariate analysis were considered as the most significant metabolites. In total, 27 metabolites for ESI+ ion mode and seven metabolites for ESI- ion mode were selected as the most significant differentiating metabolites (Supplemental Table 2).

In a comparison of differential metabolites in groups $A-B$ and $C-D$, we found similar variation tendencies. The differential metabolites are shown in Supplemental Tables 1 and 2. Most of the metabolites showing similar trends were not identified as significant differential metabolites. However, phosphohydroxypyruvic acid rates were high in groups $A-B$ and $C-D$ in ESI+ ion mode.

After selection of differential metabolites, the enriched pathways were further analyzed (Figure 3). In groups $A-B$, only glyoxylate and dicarboxylate metabolism, vitamin B6 metabolism, and pyruvate metabolism were identified as significantly enriched pathways, and two of these three pathways showed high occupancy percentages. Notably, similar to vitamin B6 metabolism in groups A-B, glycerophospholipid metabolism was also the most significantly enriched pathway in groups $C-D$ and showed the highest occupancy percentage. Phosphohydroxypyruvic acid, which is involved in glycine, serine, and threonine metabolism, was only selected in groups $C-D$.

\section{SVM analysis of blood samples delayed and enhanced postoperative recovery patients}

Identification of differential blood metabolites between the enhanced and delayed postoperative recovery groups suggested that some of the identified metabolites may have applications as biomarkers to predict whether a patient would suffer from poor postoperative recovery after surgery. Therefore, an SVM model was applied to identify the patterns among metabolites. By using these differentiated blood metabolites between group $A$ and $B$ as input features, an SVM model with 22 features was obtained (Figure 4A). An AUC score of 0.983 indicated an effective model, and the prediction accuracy reached $92.8 \%$ (Figure 4B). For samples belonging to groups $C$ and $D$, the optimal SVM model had 10 features (Figure 4D). An AUC score of 0.941 indicated an effective model, and the prediction accuracy reached $88.8 \%$ (Figure $4 \mathrm{E}$ ).

In both the A-B and C-D SVM models, there were four metabolites calculated as important features (Figure 4C and 4F). Oleamide, ubiquinone-1, acetylcholine, and oleic acid could be used to separate patients exhibiting enhanced and delayed postoperative recovery both in the PACU and at 24 hours after surgery. Thus, these metabolites could be used as potential biomarkers to predict patients' postoperative recovery state according to the SVM results. Among all these features, oleamide had the highest selected frequency, and ubiquinone-1 also ranked among the top 3 in both models.

There were 28 differential metabolites between groups A and B; 22 of these metabolites could be used as important features in the A-B SVM model. Among the nine metabolites showing similar variation trends 
compared with those in the C-D model, six were identified as SVM features. For the C-B model, 10 of 34 differential metabolites were used as important features in the SVM model, whereas five feature metabolites had similar variation trends compared with groups A-B among the 21 total metabolites.

\section{Discussion}

The recovery rate of GI function is an essential factor reflecting postoperative recovery in patients undergoing colorectal surgery. Morbidity and mortality are both influenced by postoperative care. Although GDFT guided by the ERAS program is helpful for postoperative recovery in most situations and GDFT under SVV guidance is an appropriate strategy for fluid management during and after colorectal surgery, some cases show opposite trends. Therefore, it is critical to identify methods to evaluate and predict potential GI function recovery during the early postoperative period such that countermeasures may be applied to enhance prognosis. In this study, all patients underwent colorectal surgery with GDFT under SVV guidance, and patients were grouped according to different clinical outcomes and compared based on variations in plasma metabolites.

Disease initiation and progression can also lead to changes in metabolic substances or metabolic pathways. To explore complex and dynamic living systems, omics analyses have recently been established.[16] Metabolomics, defined as "the quantitative measurement of the dynamic multiparametric metabolic response of living systems to pathophysiological stimuli or genetic modification",[17] plays an essential role in disease prediction and diagnosis. Anesthesia and surgery are both critical factors that affect postoperative GI function. Moreover, different metabolic responses among patients may influence these outcomes. Therefore, in this study, we explored differences in metabolites between patients with delayed and enhanced postoperative GI function recovery to evaluate potential differences in biological responses. Samples isolated in the PACU reflected the immediate response of metabolism after anesthesia and surgery, whereas sample collected at 24 hours after operation provided insights into more long-term changes and trends in metabolic alterations. We evaluated blood samples from 24 patients in both the delayed and enhanced postoperative recovery groups and found significant differential metabolites at both time points in both groups, allowing classification with the help of an SVM model. Finally, we identified four potential biomarkers that could be used to predict postoperative recovery both in the PACU and at 24 hours after surgery.

Differential metabolites may not only serve as biomarkers for prediction but may also suggest underlying metabolic mechanisms of GI function recovery. In our analysis of metabolic differences, 28 significant differential metabolites were identified in blood samples from patients with delayed and enhanced postoperative recovery in the PACU and 34 significant differential metabolites were identified at 24 hours after surgery. Although nine and 21 metabolites in blood samples from the PACU and at 24 hours after surgery showed similar variation trends, only phosphohydroxypyruvic acid showed significant variations. Phosphohydroxypyruvic acid is an intermediate product generated during the transformation from pyruvate to serine and is therefore related to glycine, serine, and threonine metabolism.[18] Pyruvate affects energy production, which is easily influenced by fluid management, and is reflected in 
phosphohydroxypyruvic acid levels.[19] Vitamin B6 is helpful for the postoperative recovery of patients with cerebral aneurysm,[20] and its importance in patients with colorectal cancer suggests that this compound may be beneficial in general postoperative recovery, i.e., for patients undergoing different types of surgeries. Additionally, the tricarboxylic acid (TCA) cycle is activated in energy supplementrelated processes, which can be affected by liquid management.[21]

In order to distinguish patients who were responsive to GDFT, we used an SVM model to classify patients according to the time of postoperative recovery. Using differential metabolites from samples collected in the PACU and 24 hours after surgery, we identified four metabolites that could have applications as biomarkers. Although oleamide, ubiquinone-1, and oleic acid were not related to enrichment of differential metabolite pathways, these compounds were associated with glycerophospholipid metabolism, including acetylcholine. Moreover, this pathway was highly significant pathway in groups C-D. Thus, glycerophospholipid metabolism could promote intestinal function recovery, amino acid metabolism, and the TCA cycle.[22] Amino acid metabolism promotes protein production, maintains TCA cycle anaplerosis, enhances antioxidant capacity, and stabilizes lipid membranes by suppressing glycerophospholipid metabolism. These biological processes facilitate postoperative recovery and improve outcomes.

Hardly any study has evaluated metabolic differences in patients undergoing colorectal surgery with different degrees of postoperative GI function recovery. In this study, we used SVV-guided GDFT as an intra-operative fluid strategy and explored the correlations between early postoperative metabolomic differences and postoperative intestinal function. However, we only included patients who underwent colorectal surgery, not whole abdominal surgery (e.g., cesarean section and others). Importantly, not all abdominal surgeries involve the gastrointestinal tract, and the process of intestinal function recovery may be different following different types of surgery. Colorectal surgery is known to alter the integrity of the intestinal tract, and recovery of intestinal function may mainly depend on mechanisms mediated by the intestinal tract itself. The PLS-DA model used in this study to identify significant differential metabolites showed low prediction accuracy; thus, some metabolites with the same variation tendencies in groups A$B$ and $C-D$ were not recognized as significantly different. However, the model showed good performance without excessive lifting, indicating that although we could ignore some significant differential metabolites, the selected metabolites were reliable.

\section{Conclusions}

In this study, we proposed a novel method to predict postoperative recovery of GI function using metabolomics. From only 10 metabolites isolated from blood samples collected 24 hours after surgery blood samples or 22 metabolites from blood samples collected in the PACU, we may predict the patient's possible long-term postoperative recovery condition of $\mathrm{GI}$ function. Four potential biomarkers were identified. Further studies are needed to evaluate whether these biomarkers may have therapeutic applications for promoting postoperative GI function. 


\section{Abbreviations}

GI: gastrointestinal

GDFT: Goal-directed fluid therapy

CO: cardiac output

Cl: cardiac index

SVI: stroke volume index

SVV: stroke volume variation

$\mathrm{DO}_{2}$ : oxygen delivery

SV: stroke volumes

ERAS: Enhanced Recovery After Surgery

ASA: American Society of Anesthesiologists

NYHA: New York Heart Association Functional Classification

BMI: body mass index

MAC: minimum alveolar concentration

PEEP: positive end-expiratory pressure

ECG: electrocardiograph

LC/MS: liquid chromatography-mass spectrometry

SVM: support vector machine

MBP: mean blood pressure

PACU: post-anesthesia care unit

\section{Declarations}

Ethics approval and consent to participate: The study was approved by the appropriate Institutional Review Board (IRB) and Changhai Hospital Ethics Committee (Shanghai, China; approval no. CHEC2018080) and was registered at the Chinese Clinical Trial Registry (www.chictr.org.cn; identifier: 
ChiCTR1800015899). Written informed consent was obtained from all patients or legal guardians, or the requirement for written informed consent was waived by the IRB.

Consent for publication: Patients gave consent for their personal or clinical details to be published in this study. A copy of the written consent is available for review by the Editor-in-Chief of this journal.

Availability of data and materials: The datasets used and/or analyzed during the current study are available from the corresponding author on reasonable request.

Competing interests: All authors have disclosed that they do not have any conflicts of interest.

Funding: This work was supported by the National Natural Science Foundation of China (grant nos. 81772105 to X. Deng and 81600948 to Y. Zha).

Author contributions: Dr. T. Xie and Dr. Z. Jiang analyzed the data and prepared the manuscript. Dr. T. Xie collected samples and contributed to the trial preparation and registration. Dr. C. Wen and Dr. D. Shen contributed to the data collection and patient follow-up. Dr. J. Bian and Dr. S. Liu provided constructive suggestion and contributed to patient selection, clinical intervention, and project management. Prof. X. Deng and Dr. Y. Zha designed the study and supervised the project.

\section{Acknowledgements}

We thank Dr. Shichao Pang from Shanghai Institute of Biological Sciences (SIBS), Chinese Academy of Sciences (CAS) for helpful advice and discussions regarding this manuscript.

\section{References}

1. Pearse, R.M.; Harrison, D.A.; James, P.; Watson, D.; Hinds, C.; Rhodes, A.; Grounds, R.M.; Bennett, E.D. Identification and characterisation of the high-risk surgical population in the United Kingdom. Critical care (London, England) 2006; 10, R81.

2. Mythen, M.G.; Webb, A.R. Perioperative plasma volume expansion reduces the incidence of gut mucosal hypoperfusion during cardiac surgery. Archives of surgery (Chicago, III. : 1960) 1995; 130 : 423-429.

3. Brandstrup, B.; Tonnesen, H.; Beier-Holgersen, R.; Hjortso, E.; Ording, H.; Lindorff-Larsen, K.; Rasmussen, M.S.; Lanng, C.; Wallin, L.; Iversen, L.H., et al. Effects of intravenous fluid restriction on postoperative complications: comparison of two perioperative fluid regimens: a randomized assessor-blinded multicenter trial. Annals of surgery 2003; 238: 641-648.

4. McLennan, E.; Oliphant, R.; Moug, S.J. Limited preoperative physical capacity continues to be associated with poor postoperative outcomes within a colorectal ERAS programme. Annals of the Royal College of Surgeons of England 2019; 101: 261-267.

5. Manning, M.W.; Dunkman, W.J.; Miller, T.E. Perioperative fluid and hemodynamic management within an enhanced recovery pathway. Journal of surgical oncology 2017; 116: 592-600. 
6. Taniguchi, H.; Sasaki, T.; Fujita, H.; Kobayashi, H.; Kawasaki, R.; Ogata, T.; Cho, H.; Yoshikawa, T.; Ushigome, K.; Tanaka, A., et al. Effects of goal-directed fluid therapy on enhanced postoperative recovery: An interventional comparative observational study with a historical control group on oesophagectomy combined with ERAS program. Clinical nutrition ESPEN 2018; 23: 184-193.

7. Correa-Gallego, C.; Tan, K.S.; Arslan-Carlon, V.; Gonen, M.; Denis, S.C.; Langdon-Embry, L.; Grant, F.; Kingham, T.P.; DeMatteo, R.P.; Allen, P.J., et al. Goal-Directed Fluid Therapy Using Stroke Volume Variation for Resuscitation after Low Central Venous Pressure-Assisted Liver Resection: A Randomized Clinical Trial. Journal of the American College of Surgeons 2015; 221: 591-601.

8. Rhodes, A.; Cecconi, M.; Hamilton, M.; Poloniecki, J.; Woods, J.; Boyd, O.; Bennett, D.; Grounds, R.M. Goal-directed therapy in high-risk surgical patients: a 15-year follow-up study. Intensive care medicine 2010; 36: 1327-1332.

9. Hamilton, M.A.; Cecconi, M.; Rhodes, A. A systematic review and meta-analysis on the use of preemptive hemodynamic intervention to improve postoperative outcomes in moderate and high-risk surgical patients. Anesthesia and analgesia 2011;112: 1392-1402

10. Berkenstadt, H.; Margalit, N.; Hadani, M.; Friedman, Z.; Segal, E.; Villa, Y.; Perel, A. Stroke volume variation as a predictor of fluid responsiveness in patients undergoing brain surgery. Anesthesia and analgesia 2001; 92: 984-989.

11. Varadhan, K.K.; Neal, K.R.; Dejong, C.H.; Fearon, K.C.; Ljungqvist, O.; Lobo, D.N. The enhanced recovery after surgery (ERAS) pathway for patients undergoing major elective open colorectal surgery: a metaanalysis of randomized controlled trials. Clinical nutrition (Edinburgh, Scotland) 2010; 29: 434-440.

12. Fearon, K.C.; Ljungqvist, O.; Von Meyenfeldt, M.; Revhaug, A.; Dejong, C.H.; Lassen, K.; Nygren, J.; Hausel, J.; Soop, M.; Andersen, J., et al. Enhanced recovery after surgery: a consensus review of clinical care for patients undergoing colonic resection. Clinical nutrition (Edinburgh, Scotland) 2005; 24: 466-477.

13. Bujak, R.; Struck-Lewicka, W.; Markuszewski, M.J.; Kaliszan, R. Metabolomics for laboratory diagnostics. Journal of pharmaceutical and biomedical analysis 2015; 113: 108-120.

14. Srinivasa, S.; Taylor, M.H.; Singh, P.P.; Yu, T.C.; Soop, M.; Hill, A.G. Randomized clinical trial of goaldirected fluid therapy within an enhanced recovery protocol for elective colectomy. The British journal of surgery $2013 ; 100: 66-74$.

15. The Lancet, H. Updates on blood transfusion guidelines. Lancet Haematol 2016; 3: e547.

16. Goh, K.I.; Cusick, M.E.; Valle, D.; Childs, B.; Vidal, M.; Barabasi, A.L. The human disease network. Proceedings of the National Academy of Sciences of the United States of America 2007; 104: 86858690.

17. Nicholson, J.K.; Lindon, J.C.; Holmes, E. 'Metabonomics': understanding the metabolic responses of living systems to pathophysiological stimuli via multivariate statistical analysis of biological NMR spectroscopic data. Xenobiotica; the fate of foreign compounds in biological systems 1999; 29: 1181-1189. 
18. Kalhan, S.C.; Uppal, S.O.; Moorman, J.L.; Bennett, C.; Gruca, L.L.; Parimi, P.S.; Dasarathy, S.; Serre, D.; Hanson, R.W. Metabolic and genomic response to dietary isocaloric protein restriction in the rat. The Journal of biological chemistry 2011; 286: 5266-5277.

19. Guo, J.; Zhang, J.; Luo, X.; Luo, W.; Lin, C.; Zhang, K.; Ji, Y. Effects of ethyl pyruvate on cardiac function recovery and apoptosis reduction after global cold ischemia and reperfusion. Experimental and therapeutic medicine 2014; 7: 1197-1202.

20. Chen, X.; Lei, T. Glycerol fructose combined with vitamin B6 is beneficial to postoperative recovery of patients with cerebral aneurysm. Experimental and therapeutic medicine 2018; 16: 236-240.

21. Olivson, A.; Berman, E.; Houminer, E.; Borman, J.B.; Merin, G.; Karck, M.; Haverich, A.; Chisin, R.; Schwalb, H. Glucose metabolism, energetics, and function of rat hearts exposed to ischemic preconditioning and oxygenated cardioplegia. Journal of cardiac surgery 2002; 17: 214-225.

22. Das, U.N. Essential fatty acids: biochemistry, physiology and pathology. Biotechnology journal 2006; 1: 420-439.

\section{Tables}


Table 1

Characteristics of the study population
Enhanced $(n=24)$
Delayed $(n=24)$
$P$ value

\section{Baseline}

Age (years)

$56.04 \pm 5.58$

$53.88 \pm 8.41$

0.2

Female sex

9

9

1

BMI

$22.67 \pm 2.06$

$22.5 \pm 2.22$

0.772

ASA (I/II/III)

$6 / 16 / 2$

$3 / 20 / 1$

\section{Comorbidities}

Hypertension

3

0

0.234

\section{Type of surgery}

Anterior resection of rectum

9

6

0.534

Left hemicolectomy

4

6

0.724

Right hemicolectomy

3

4

$>0.999$

Transverse colectomy

4

3

$>0.999$

Other

4

5

$>0.999$

Intra-operative data

Operation duration (min)

$142.5 \pm 54.39$

$133.9 \pm 45.34$

0.562

SVV (before surgery)

$7.96 \pm 1.88$

$8.21 \pm 1.96$

0.651

SVV (after surgery)

$6.29 \pm 1.65$

$6.42 \pm 1.74$

0.803

MBP (before surgery)

$77.67 \pm 7.11$

$75.63 \pm 8.81$

0.381

MBP (after surgery)

$83.46 \pm 7.84$

$86.63 \pm 10.64$

0.247

Intra-operative fluids

$1505 \pm 468.4$

$1520 \pm 497.7$

0.915

$24 \mathrm{~h}$ peri-operative fluids

$3403 \pm 622$

$3415 \pm 165.2$

0.956

\section{Outcomes}

Flatus time

Defecation

Feeding time
$1.83 \pm 0.38$

$3.46 \pm 1.50$

$3.17 \pm 1.31$
$3.63 \pm 1.35$

$5.13 \pm 1.39$

$4.71 \pm 2.65$
$<0.001$

$<0.001$

0.014

Abbreviations: BMI, body mass index; ASA, American Society of Anesthesiologists classification; CAD, coronary artery disease; SVV, stroke volume variation; MBP, mean blood pressure 


\begin{tabular}{|llll|}
\hline & Enhanced $(\mathbf{n}=\mathbf{2 4})$ & Delayed $(\mathbf{n}=\mathbf{2 4})$ & $P$ value \\
\hline Hospital stay (days) & $9.58 \pm 1.95$ & $10 \pm 3.02$ & 0.572 \\
\hline Complications & & & \\
\hline lleus & 1 & 2 & $>0.999$ \\
\hline Fistula & 0 & 1 & $>0.999$ \\
\hline Incision infection & 2 & 2 & $>0.999$ \\
\hline Anemia & 0 & 5 & 0.049 \\
\hline Postoperative nausea & 4 & 7 & 0.049 \\
\hline Postoperative vomiting & 1 & 4 & 0.0028 \\
\hline $\begin{array}{l}\text { Abbreviations: BMI, body mass index; ASA, American Society of Anesthesiologists classification; CAD, } \\
\text { coronary artery disease; SVV, stroke volume variation; MBP, mean blood pressure }\end{array}$ \\
\hline
\end{tabular}

\section{Figures}

A

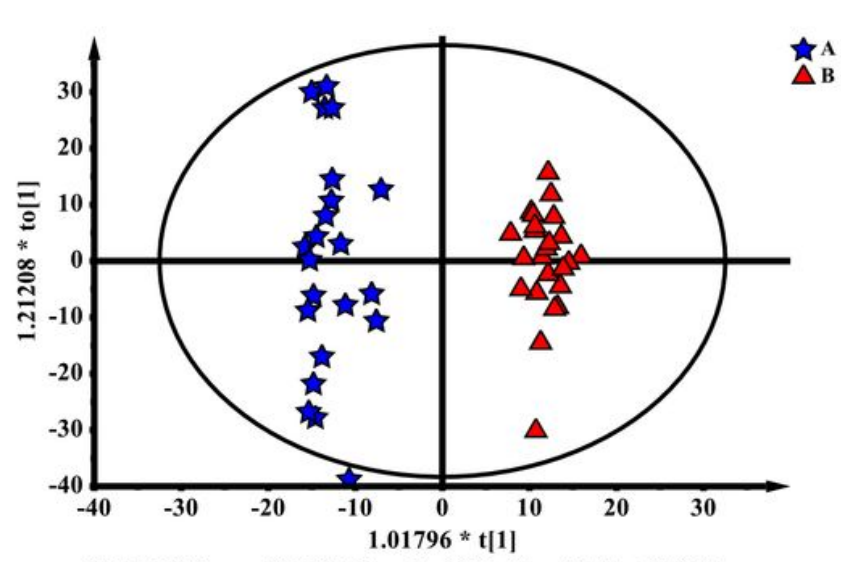

R2X $\mid 1]=0.0346 \quad R 2 X \mid X$ Side Comp. 1$]=0.0481$ Ellipse: Hotelling's T2 (95\%)

c

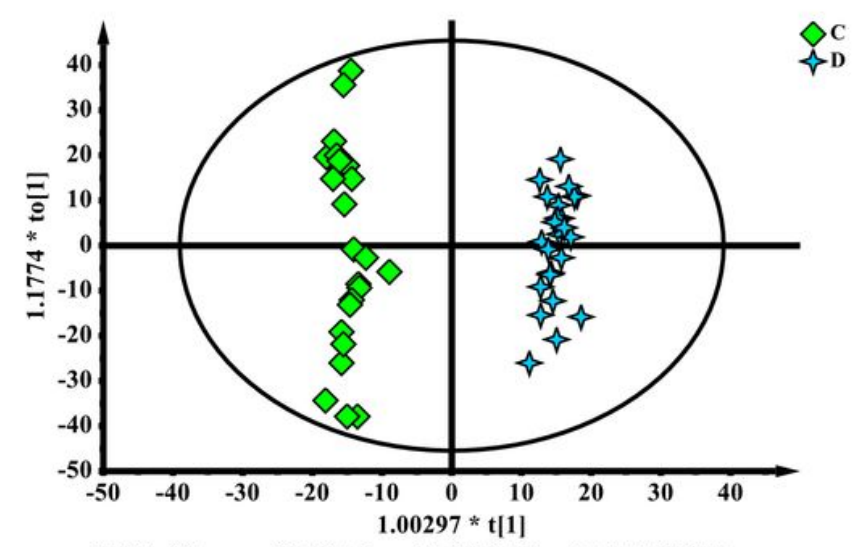

R2X [1] $=0.05 \quad$ R2X|XSide Comp. 1] $=0.0679$ Ellipse: Hotelling's T2 (95\%)
B

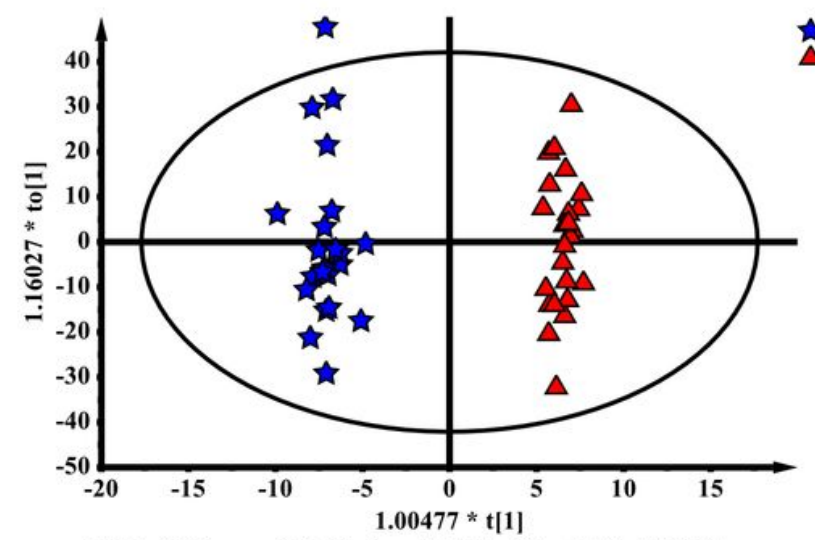

R2X $[1]=0.0251 \quad$ R2X|XSide Comp. 1] $=0.142$ Ellipse: Hotelling's T2 (95\%)

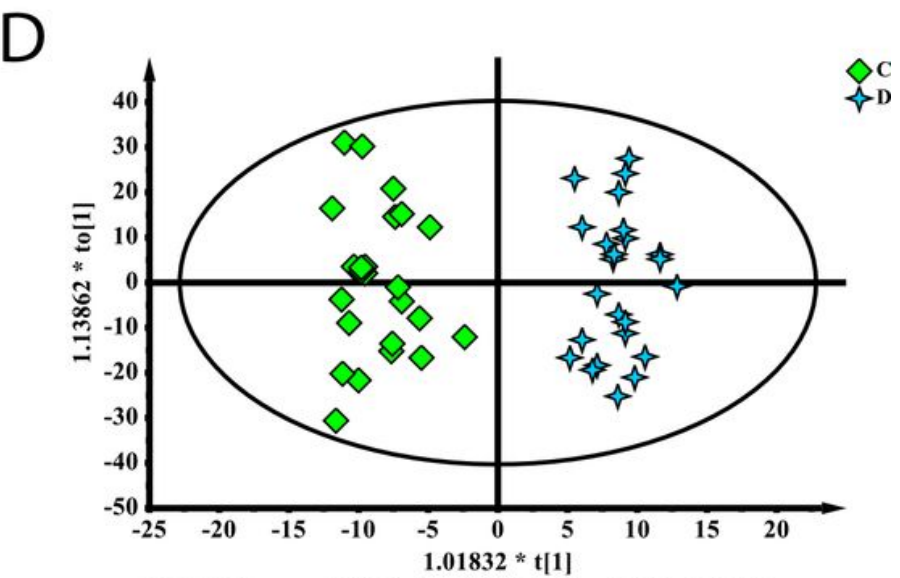

R2X $[1]=0.042 \quad R 2 X \mid X$ Side Comp. 1] $=0.131$ Ellipse: Hotelling's T2 (95\%) 
Figure 1

A. Partial least squares discriminant analysis of blood metabolomics data from delayed and enhanced postoperative recovery groups. Blood metabolites were used to distinguish patients with different postoperative recovery outcomes.

A

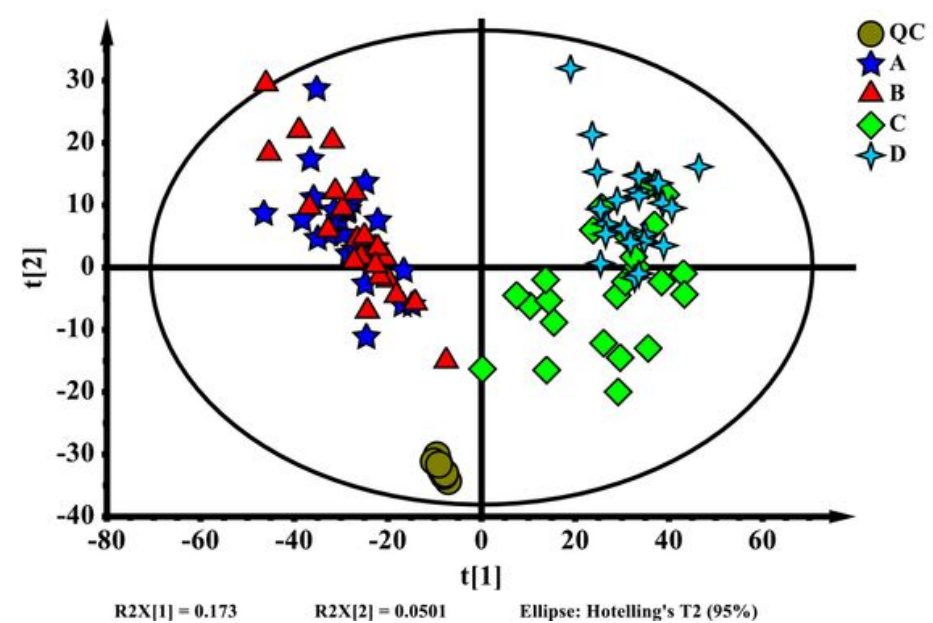

B

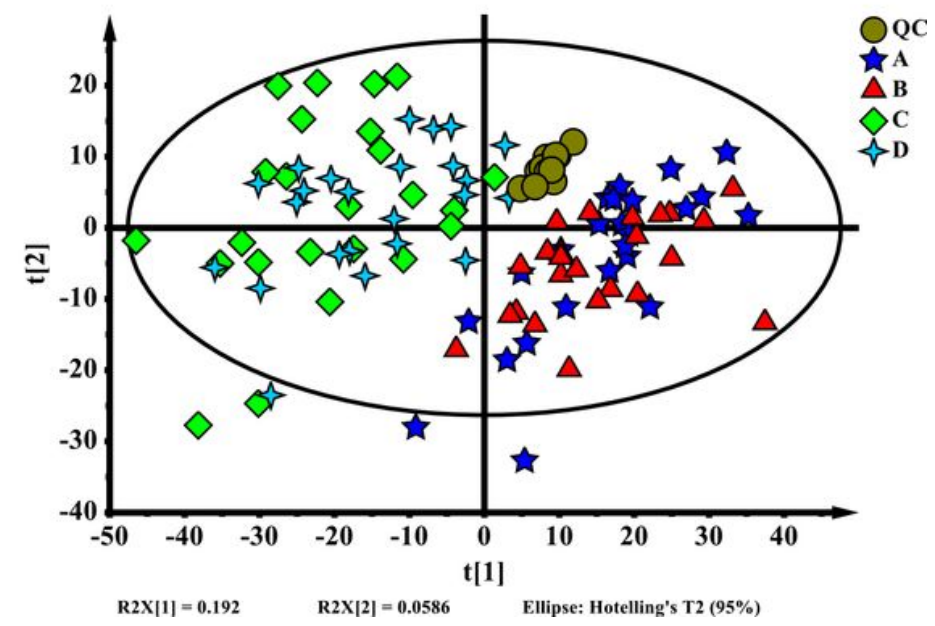

Figure 2

A. Orthogonal partial least squares discriminant analysis (OPLS-DA) score plot of all samples (ESI+). B. OPLS-DA score plot of all samples (ESI-). 


\section{Pathway analysis of differential metabolites}

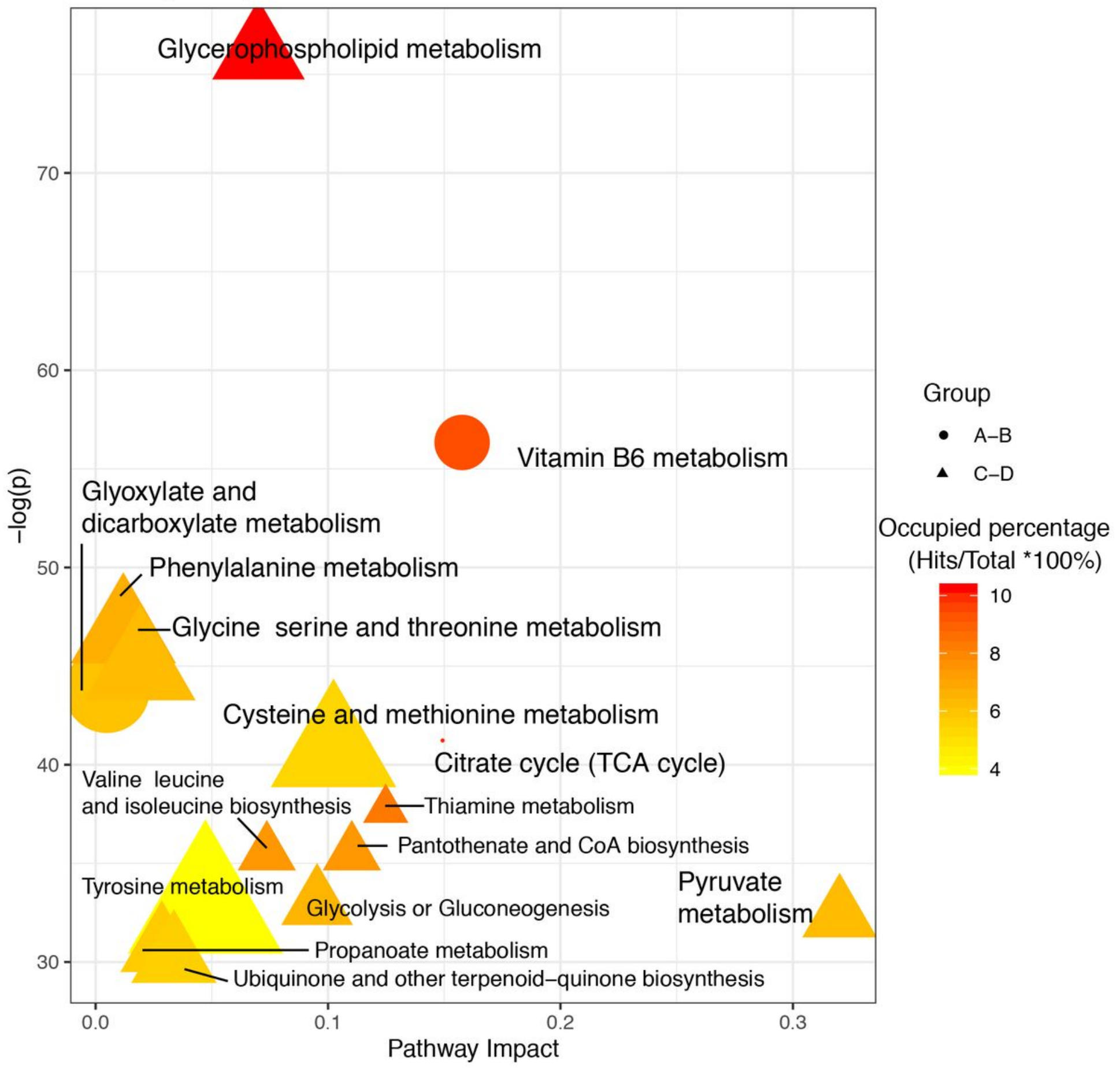

\section{Figure 3}

Pathway analysis of altered metabolites isolated from patients with delayed and enhanced postoperative recovery in the postanesthesia care unit and at $24 \mathrm{~h}$ after surgery. The $\mathrm{X}$-axis represents the pathway impact, and the $Y$-axis represents the - $\log (\mathrm{p})$. Node size indicates metabolites in a specific pathway, and colors indicate the occupied percentage (the hit metabolites occupying a percentage of all metabolites in the whole pathway). Yellow to red represents high to low. The shape of the node represents differential metabolites in different groups. 
A

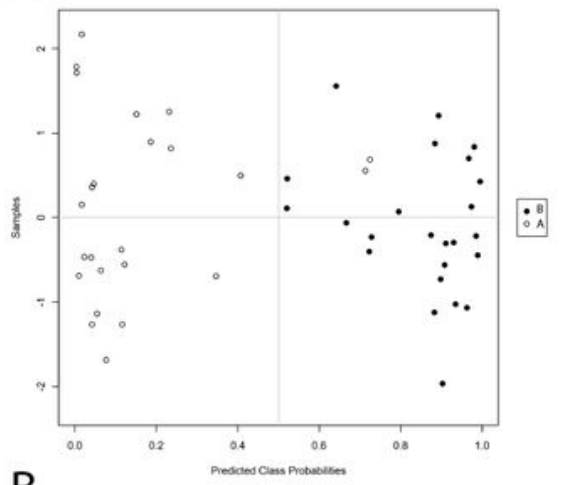

B
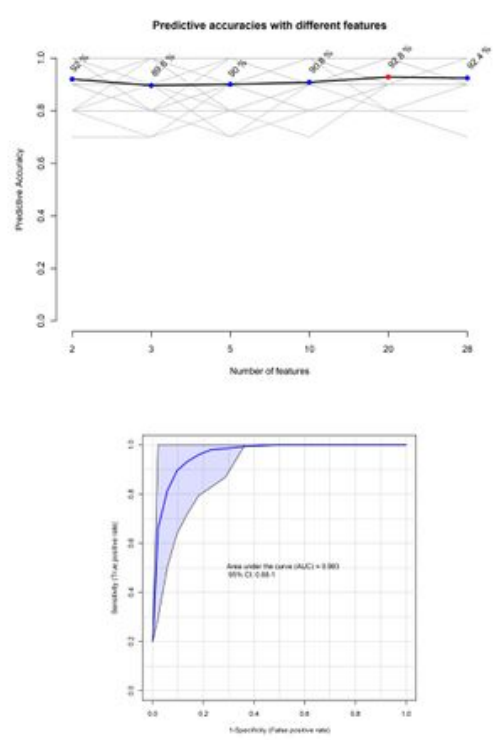

C

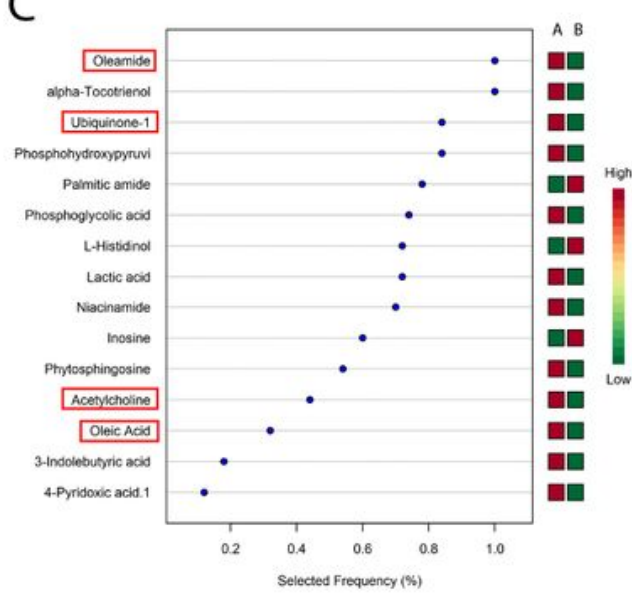

D
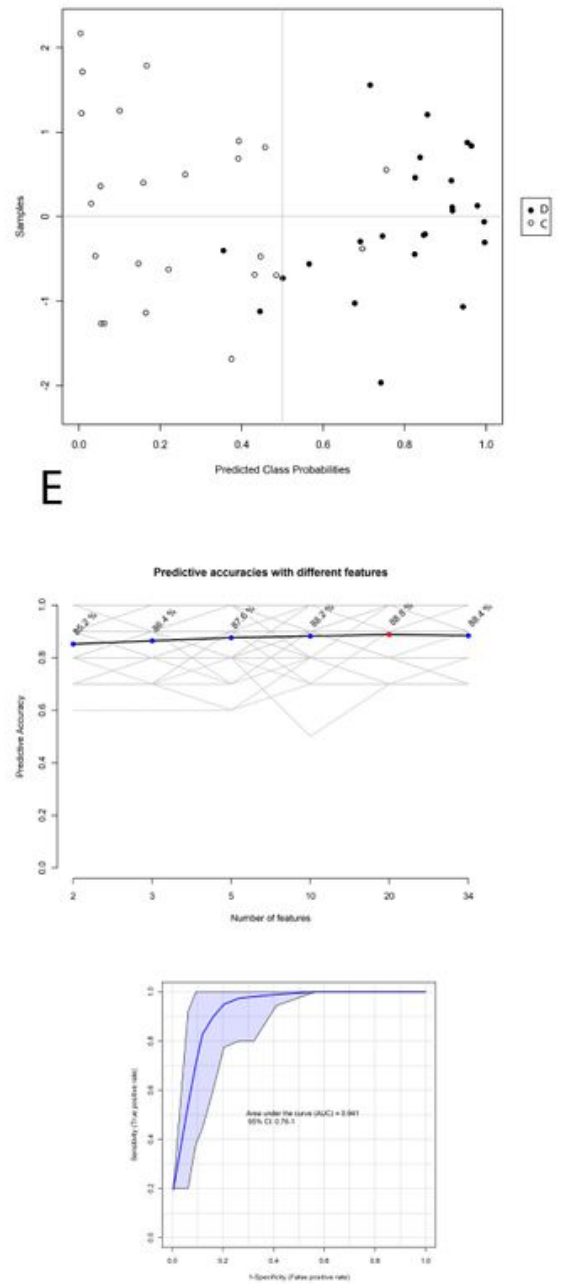

F

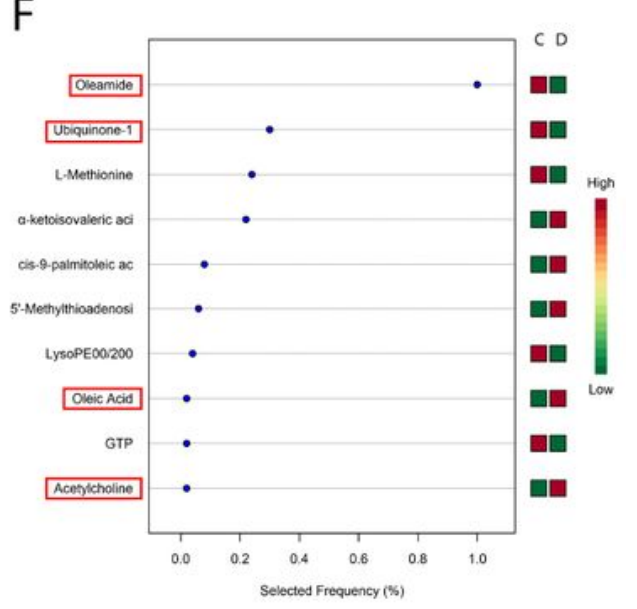

\section{Figure 4}

Support vector machine (SVM) analysis of metabolites in blood samples from patients in the in postanesthesia care unit and at $24 \mathrm{~h}$ after surgery in patients with delayed and enhanced postoperative recovery. A. SVM classification of samples in groups A and B. B. Specificity, sensitivity, and predictive accuracies of the A-B SVM model. C. Features' importance ranking of the A-B SVM model. D. SVM classification of samples belonging to groups C and D. E. Specificity, sensitivity, and predictive accuracies 
of the C-D SVM model. F. Features' importance ranking of the C-D SVM model. The red cube represents higher importance, whereas the green cube represents lower importance in the SVM model.

\section{Supplementary Files}

This is a list of supplementary files associated with this preprint. Click to download.

- SupplementaryFigureLegends.docx

- SupplementaryFigureLegends.docx

- SupplementaryFigureLegends.docx

- SupplementaryFigure1.pdf

- SupplementaryFigure1.pdf

- SupplementaryFigure1.pdf

- SupplementalTable2.docx

- SupplementalTable2.docx

- SupplementalTable2.docx

- SupplementalTable1.docx

- SupplementalTable1.docx

- SupplementalTable1.docx 